\title{
Keen, S. (2017). Pouvons-nous éviter une autre crise financière? Paris, France : Les liens qui libèrent, 160 p.
}

\author{
Marc-André Morency ${ }^{\mathbf{a}}$
}
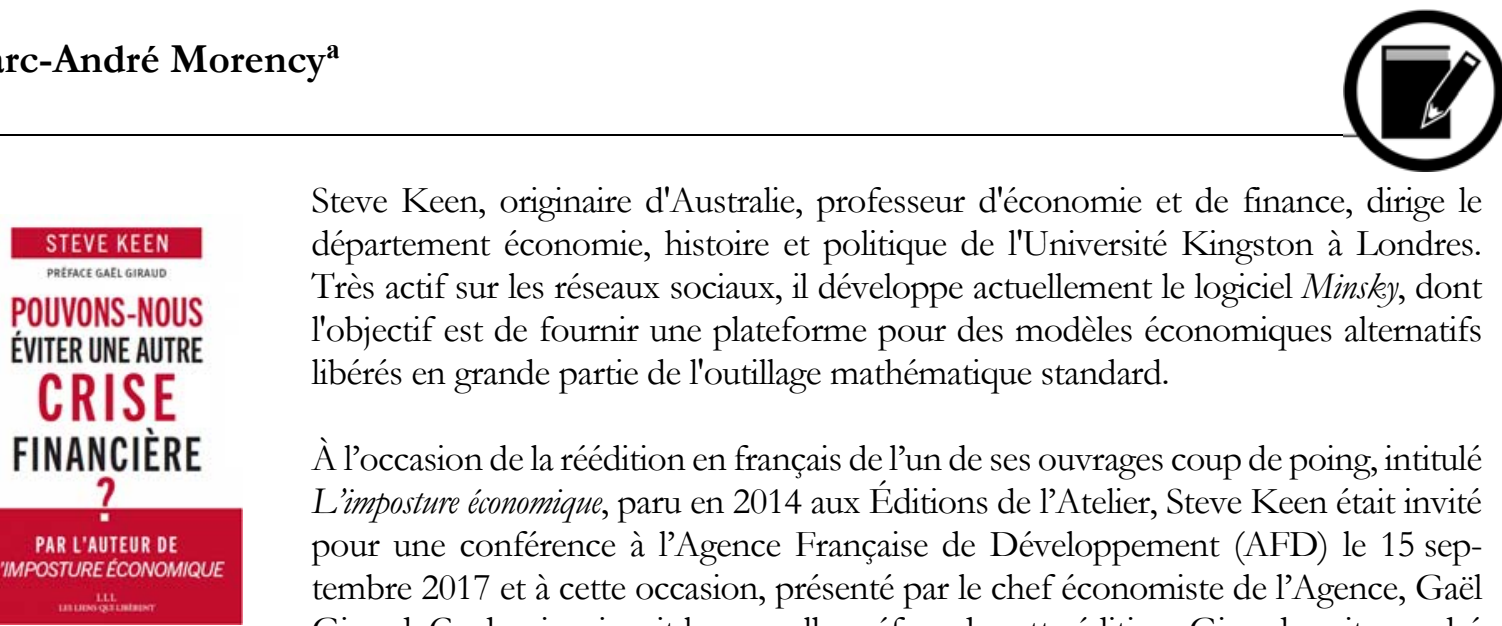

Steve Keen, originaire d'Australie, professeur d'économie et de finance, dirige le département économie, histoire et politique de l'Université Kingston à Londres. Très actif sur les réseaux sociaux, il développe actuellement le logiciel Minsky, dont l'objectif est de fournir une plateforme pour des modèles économiques alternatifs libérés en grande partie de l'outillage mathématique standard.

À l'occasion de la réédition en français de l'un de ses ouvrages coup de poing, intitulé L'imposture économique, paru en 2014 aux Éditions de l'Atelier, Steve Keen était invité pour une conférence à l'Agence Française de Développement (AFD) le 15 septembre 2017 et à cette occasion, présenté par le chef économiste de l'Agence, Gaël Giraud. Ce dernier signait la nouvelle préface de cette édition. Giraud avait encadré la traduction et préfacé la première édition française de l'ouvrage. Ces économistes font partie de ceux, de plus en plus nombreux, qui font la promotion d'une vision critique de l'économisme néoclassique dominant actuellement les académies, les journaux spécialisés, les instances de la recherche subventionnée...

Au cœur de la discussion : les lacunes de cette théorie dominante actuellement en économique, la théorie dite " néo-classique », extrêmement conservatrice dans ses perspectives et impacts dans la gouvernance de l'économie, et qui pourtant fait l'impasse sur l'épargne et la dette privée, la croissance, la monnaie, les inégalités croissantes, le rôle central de l'énergie dans l'économie, etc. Ces lacunes sont, selon Keen et Giraud, responsables de l'incapacité des économistes non critiques à surveiller les facteurs de crise, l'endettement privé ou public, à juguler durablement les crises que connaît à répétition le capitalisme alors que le crédit joue un rôle majeur dans les équilibres macro-économiques, incapacité et donc à recommander les approches qui permettraient aux États de mieux gouverner, soit individuellement, soit collectivement à l'échelle des phénomènes contemporains.

Les Médicis avaient compris le caractère peu fiable des États et refusaient de leur prêter. Aujourd'hui, c'est tout le contraire, on prête à l'État endetté, aux personnes privées endettées, mais avec extrême réticence aux entrepreneurs et entreprises. La dette privée a explosé au point qu'elle menace dans ses fondements l'avenir du système tout entier à l'échelon global. Steve Keen a été l'un des quelques économistes au monde à avoir alerté quant à l'imminence de la crise des subprimes et d'autres papiers commerciaux déclenchée en 2007-2008, crise dont nous ne sommes pas encore sortis. Il dénonce la faiblesse de la règlementation qui permet actuellement aux banques de recourir aux mêmes instruments délétères que dans la période de "grande modération » soit la titrisation et le crédit sans véritables limites. La situation actuelle est qualifiée de normale alors même que de nombreux pays, sinon tous, se trouvent engagés dans une course sans frein à la croissance basée sur le crédit privé, pendant que les entreprises peinent à trouver du financement pour leur développement.

Keen propose une critique radicale de l'argumentaire de la théorie néoclassique, laquelle joue depuis 1980, le rôle d'une théorie dominante dans le monde des économistes. Les gardiens de la Réserve fédérale des ÉtatsUnis (FED), Alan Greenspan et Ben Bernanke, laissent faire la dérive initiée par le Président Clinton, une

a Professeur retraité, sociologue, Université du Québec à Chicoutimi 
création monétaire débridée, quitte à policer un cas extrême en 2008 (dissolution forcée de la banque d'investissement Lehman Brothers) et à organiser la fusion des géants responsables de la crise. Hank Paulsen se chargera de dépenser les 700 milliards US du secteur public appelé à la rescousse.

Le constat de Steve Keen est sans appel : si les économistes continuent de délaisser la prise en compte d'éléments aussi importants que la création monétaire ou la dette, ils ne pourront pas empêcher l'émergence d'autres crises économiques et encore moins les anticiper. Fils d'un père directeur de banque, Keen a pu trouver là une source d'inspiration non négligeable, qu'il a complétée par une attentive relecture de l'économiste critique américain Hyman Minsky (1919-1996). Minsky, c'est, en passant, le nom qu'il a donné à un simulateur économique disponible en ligne sur Internet. Il s'agit d'un logiciel qui, sous forme graphique, permet de modéliser les questions macroéconomiques sans recourir aux raccourcis issus de la micro-économique. Ce logiciel est tenu à jour, et se trouve très bien illustré et commenté. Il devrait plaire à tous ceux qui aimeraient développer leur raisonnement en économique, mais sont rebutés par l'appareillage mathématique standard qui le rend si abscons pour les non-professionnels, sinon les professionnels eux-mêmes.

Comme Minsky, Steve Keen interroge les fondements de l'économie dominante pour mieux les déconstruire. En particulier, il dénonce la transposition des abstractions de la microéconomie (Jevons, Marshall) en modèles macroéconomiques non émergents, non systémiques qui négligent les questions monétaires, les formes de crédit, le rôle des banques et des banquiers centraux. Ce sont des modèles incapables d'anticiper les crises à répétition que produit le capitalisme depuis ses débuts. Inutile de dire que l'on se trouve encore bien loin d'aborder le système-monde qui impose de par son existence même des lois que l'abstraction mathématique de la perspective micro-économique n'arrive pas à représenter. Keen salue au passage le rôle de la Banque des Règlements Internationaux (BRI) qui a entrepris de suivre de très près les dérives du crédit à l'échelle mondiale et de lancer les premiers cris d'alarme officiels avant la crise de 2007-8. Keen trouve de nombreux indicateurs utiles dans les travaux du BRI ainsi que dans les données officielles du commerce américain; celles-ci offrent de l'information pertinente depuis les années 1830.

Steve Keen pose les bases d'une nouvelle manière de penser l'économie, en raisonnant à partir de grands agrégats monétaires et financiers, indicateurs des formes de crédit, du revenu partagé (retour en force de l'analyse de la distribution des revenus en trois classes bien distinctes), du niveau d'emploi ou d'inoccupation, du PIB, des pratiques financières à la Ponzi des milieux de la banque, les ressources ponctionnées des puissances publiques à l'occasion des « crises », etc.

Son analyse de la fabrique des «zombies » de la dette privée explique en particulier le cas japonais et les difficultés actuelles de la Chine. Ce ne sont que des aspects particuliers de l'endettement insoutenable que connait le monde, lequel promet, selon Steve Keen, un dérapage global à l'image de ce que connaît le Japon depuis plus de 20 ans ${ }^{1}$.

\section{NOTES}

1 Nous nous permettons de préciser que, selon le Fonds monétaire international (FMI), l'ensemble des dettes publiques et privées à l'échelle mondiale atteignait un sommet historique de 184000 milliards \$US en 2017. L'essentiel de cette dette est le fait des pays riches, les États-Unis comptant à eux seuls pour presque 50000 milliards et le Japon, pour presque

20000 milliards. Leur poids dans le total va toutefois en diminuant, les économies émergentes ayant commencé, elles aussi, à s'endetter de plus en plus, menées par la Chine, dont la dette dépasse 30000 milliards. Fonds monétaire international (octobre 2018). Rapport sur la stabilité financière dans le monde. Repéré à https://www.imf.org/fr/ Publications/GFSR/Issues/2018/09/25/Global-Financial-Stability-Report-October-2018

\section{RÉFÉRENCES}

Keen, S. (2014). L'imposture économique. Paris, France : Éditions de l'Atelier. 